OPEN ACCESS

Edited by:

Nils Helge Schebb, University of Wuppertal, Germany

Reviewed by:

Hartmut Kuhn,

Charité Medical University of Berlin,

Germany

Joan Clària,

Hospital Clínic de Barcelona, Spain

Ameer Taha,

University of California, Davis, United States

${ }^{*}$ Correspondence:

Ryan G. Snodgrass

snodgrass@biochem.uni-frankfurt.de

Specialty section:

This article was submitted to Inflammation Pharmacology,

a section of the journal

Frontiers in Pharmacology

Received: 11 April 2019

Accepted: 05 June 2019

Published: 04 July 2019

Citation:

Snodgrass $R G$ and Brüne $B$ (2019) Regulation and Functions of 15-Lipoxygenases in Human Macrophages.

Front. Pharmacol. 10:719. doi: 10.3389/fphar.2019.00719

\section{Regulation and Functions of 15-Lipoxygenases in Human Macrophages}

\author{
Ryan G. Snodgrass * and Bernhard Brüne \\ Faculty of Medicine, Institute of Biochemistry I, Goethe-University Frankfurt, Frankfurt, Germany
}

Lipoxygenases (LOXs) catalyze the stereo-specific peroxidation of polyunsaturated fatty acids (PUFAs) to their corresponding hydroperoxy derivatives. Human macrophages express two arachidonic acid (AA) 15-lipoxygenating enzymes classified as ALOX15 and ALOX15B. ALOX15, which was first described in 1975, has been extensively characterized and its biological functions have been investigated in a number of cellular systems and animal models. In macrophages, ALOX15 functions to generate specific phospholipid (PL) oxidation products crucial for orchestrating the nonimmunogenic removal of apoptotic cells (ACs) as well as synthesizing precursor lipids required for production of specialized pro-resolving mediators (SPMs) that facilitate inflammation resolution. The discovery of ALOX15B in 1997 was followed by comprehensive analyses of its structural properties and reaction specificities with PUFA substrates. Although its enzymatic properties are well described, the biological functions of ALOX15B are not fully understood. In contrast to ALOX15 whose expression in human monocyte-derived macrophages is strictly dependent on Th2 cytokines IL-4 and IL-13, ALOX15B is constitutively expressed. This review aims to summarize the current knowledge on the regulation and functions of ALOX15 and ALOX15B in human macrophages.

\section{Keywords: lipoxygenase, macrophage, lipid mediator, inflammation, immunity, cholesterol}

\section{INTRODUCTION}

Lipoxygenases (LOXs) are non-heme iron-containing dioxygenases that catalyze the stereo-specific peroxidation of polyunsaturated fatty acids (PUFAs) containing one or more 1,4-cis,cis pentadiene moieties to the corresponding hydroperoxy derivatives (Kuhn et al., 2018). In mammals, LOX enzymes are expressed in numerous cell types including epithelial, endothelial, and immune cells and are involved in various functions including skin barrier formation, cell differentiation, and immunity (Kuhn et al., 2015). The human genome contains six functional LOX genes (ALOX15,

\footnotetext{
Abbreviations: AA, arachidonic acid; AC, apoptotic cell; ALOX5, arachidonate 5-lipoxygenase; ALOX15, arachidonate 15-lipoxygenase; ALOX15B, arachidonate 15-lipoxygenase type B; AMPK, AMP-activated protein kinase; CE, cholesteryl ester; DHA, docosahexaenoic acid; EPA, eicosapentaenoic acid; HEPA, hydroxyeicosapentaenoic acid; HETE, hydroxyeicosatetraenoic acid; HDHA, hydroxydocosahexaenoic acid; HIF-1a, hypoxia-inducible factor 1 $\alpha$; HNP, normal human prostate; HODE, hydroxyoctadecadienoic acid; LA; linoleic acid; LDL, low-density lipoprotein; LOX, lipoxygenase; LPS, lipopolysaccharide; LX, lipoxin; oxCE, oxidized CE; oxLDL, oxidized LDL; oxPL, oxidized phospholipid; PC, phosphatidylcholine; PCa, prostate cancer; PE, phosphatidylethanolamine; PEBP1, phosphtidylethanolamine-binding protein 1; PL, phospholipid; PUFA, polyunsaturated fatty acid; Rv, resolvin; SPM, specialized pro-resolving mediator; STAT, signal transducer and activator of transcription.
} 
ALOX15B, ALOX12, ALOX12B, ALOX5, and ALOXE3) each encoding a distinct LOX enzyme (Ivanov et al., 2015). All mammalian LOXs are single polypeptide chain proteins that fold into a two-domain structure (Kuhn et al., 2015). The smaller $\mathrm{N}$-terminal domain consists of several parallel and anti-parallel $\beta$-sheets that regulate activity and facilitate membrane binding. The C-terminal catalytic domain consists of several helices and contains the catalytic non-heme iron localized in the putative substrate-binding pocket.

Macrophages are versatile immune cells strategically positioned throughout body tissues (Varol et al., 2015). They are endowed with a broad functional repertoire of sensors allowing them to respond to a variety of environmental cues and acquire diverse but specialized functional phenotypes crucial for orchestrating initiation, progression, and the resolution of inflammation (Murray et al., 2014). In addition to classically activated pro-inflammatory macrophages and anti-inflammatory macrophages, resolution-phase macrophages are immune regulatory, endowed with aspects of both pro-inflammatory and anti-inflammatory macrophages (Stables et al., 2011). Resolution-phase macrophages are highlighted by the strong up-regulation of arachidonate 15-lipoxygenase (ALOX15), a key enzyme involved in the synthesis of specialized pro-resolving mediators (SPMs) including lipoxins (LXs), resolvins (Rvs), protectins, and maresins that facilitate inflammation resolution (Buckley et al., 2014). For this reason, ALOX15 has attracted much attention for its role in contributing to active resolution of the inflammatory process. Interestingly enough, ALOX15 is not an exclusive 15-lipoxygenating enzyme. Arachidonate 15-lipoxygenase type B (ALOX15B), which is also expressed in human macrophages (Wuest et al., 2012), catalyzes the stereospecific peroxidation of PUFAs to the same hydroperoxy derivatives as ALOX15 (Kutzner et al., 2017). Furthermore, in contrast to ALOX15 whose expression is restricted to certain macrophage phenotypes, ALOX15B is constitutively expressed in human macrophages (Wuest et al., 2012; Snodgrass et al., 2018). Knockout experiments suggest that the various mammalian LOX enzymes exhibit different biological functions. In this respect, the multiplicity of 15-lipoxygenase enzymes in human macrophages likely does not reflect functional redundancy but rather specialized biological functions. This review aims to summarize our cumulative understanding of the roles of ALOX15 and ALOX15B in human macrophages.

\section{ACTIVITY OF HUMAN ALOX15 AND ALOX15B ENZYMES}

LOXs oxygenate PUFAs including linoleic acid (LA; C18: $\Delta 2$, $\omega-6)$, alpha-linolenic acid (ALA; C18: $\Delta 3, \omega-3)$, gammalinolenic acid (GLA; C18: $\Delta 3, \omega-6$ ), eicosapentaenoic acid (EPA; $\mathrm{C} 20: \Delta 5, \omega-3)$, and docosahexaenoic acid (DHA; C22: $\Delta 6$, $\omega-3)$ to their corresponding hydroperoxy derivative but were traditionally classified with respect to their positional specificity of arachidonic acid (AA; C20: $\Delta 4, \omega-6$ ) oxygenation (Kuhn et al., 2015). Following the discovery of its rabbit ortholog in immature red blood cells (Schewe et al., 1975), human ALOX15 was reported to oxygenate AA primarily at carbon 15 producing 15-hydroxyeicosatetraenoic (HETE), hence its name (Sigal et al., 1990). Subsequent studies lead to the identification of a second human LOX capable of oxygenating AA at carbon 15, which was given the name ALOX15B (Brash et al., 1997). To differentiate between genes and proteins in this review, we use traditional formatting conventions in which gene symbols are italicized while symbols for proteins are not italicized. Symbols composed of uppercase letters refer to human genes and proteins while lowercase letters refer to non-human and murine genes and proteins. Although LOX nomenclature appears straightforward, the classification system fails to take into consideration the extent of each enzyme's reaction specificity, which can lead to confusion and misunderstanding (Table 1).

For example, oxygenation of AA by human ALOX15 and orthologs of higher primates including chimpanzees produce predominately 15-HETE and small amounts of 12-HETE while murine Alox15 and orthologs from mammals ranked lower in evolution, including rats and pigs, produce only small amounts of 15-HETE but primarily 12-HETE (Adel et al., 2016; Kuhn et al., 2018). In contrast to ALOX15, ALOX15B exclusively produces 15-HETE while its murine ortholog Alox15b (also named Alox8 which is encoded by the Alox8 gene) is an 8-lipoxygenating enzyme producing 8-HETE from AA substrate (Ivanov et al., 2015). Furthermore, the reaction specificities of 15-LOXs with omega-3 PUFAs are variable and cannot be predicted or inferred from the product pattern of AA oxygenation (Kutzner et al., 2017). With increasing diversity of the LOX family, a sequencerelated classification system based on enzyme characteristics including the degree of amino acid sequence conservation, genomic organization, catalytic similarity, and evolutionary relatedness rather than the traditional AA specificity-based classification system has been suggested (Ivanov et al., 2015).

Although SPMs are widely recognized for their role in resolving inflammation and stimulating tissue regeneration, the monohydroxy fatty acid products of the 15-lipoxygenase reaction also exhibit biological activity and are thought to serve various physiological functions (Ivanov et al., 2015). AA-derived 15- and 12-HETE exhibit both pro- and antiinflammatory effects (Singh and Rao, 2019). 15-HETE has been reported to bind and activate peroxisome proliferator-activated receptor $\gamma(\operatorname{PPAR} \gamma)$ in both human and murine macrophages (Huang et al., 1999) while 12-HETE was shown to activate

TABLE 1 | Human 15-lipoxygenase genes and their murine orthologs.

\begin{tabular}{ccll}
\hline & $\begin{array}{c}\text { Gene } \\
\text { symbol }\end{array}$ & \multicolumn{1}{c}{ Gene name } & \multicolumn{1}{c}{$\begin{array}{c}\text { Commonly used } \\
\text { synonyms }\end{array}$} \\
\hline Human & ALOX15 & Arachidonate & $15-L O X, 15-L O X-1$, \\
& & 15-lipoxygenase & $12 / 15-L O X, 15-L O$ \\
Mouse & \multirow{2}{*}{ AloX15 } & Arachidonate & $15-L O X, 12 / 15-L O, 12 / 15-$ \\
& & 15-lipoxygenase & LOX, 15-LO \\
Human & \multirow{2}{*}{ ALOX15B } & Arachidonate & $15-L O X-2,15-L O X 2$, \\
Mouse & \multirow{2}{*}{ AloX8 } & 15-lipoxygenase type B & 15-LOX-B \\
& & Arachidonate & Alox15b, 8-LOX \\
& & 8-lipoxygenase &
\end{tabular}


extracellular signal-regulated kinase 1/2 (ERK1/2) and nuclear factor kappa-light-chain-enhancer of activated B cells (NF- $\kappa$ B) via G protein-coupled receptor 31 (GPR31) (Guo et al., 2011). Human macrophages incubated with 12- and 15-HETE showed increases in LPS-induced gene expression (Namgaladze et al., 2015) while ALOX15 was reported to induce the production of pro-inflammatory cytokine IL-12 in macrophages (Li et al., 2013). LA-derived 13-hydroxyoctadecadienoic acid (HODE) was shown to both activate PPAR $\gamma$ and suppress PPAR $\delta$ activity (Huang et al., 1999; Shureiqi et al., 2003; Zuo et al., 2006) as well as induce oxidative stress, ER stress, and apoptosis in murine hepatoma cells (Zhang et al., 2017). Although 15- and 12-hydroxyeicosapentaenoic acid (HEPA) derived from EPA and 17- and 14-hydroxy-DHA (HDHA) generated from DHA function as intermediate precursors for the biosynthesis of Rvs, protectins, and maresins (Buckley et al., 2014), biological functions of these individual omega-3 monohydroxy fatty acids remain limited. Recently, insight into the monohydroxylated fatty acid product patterns catalyzed by human ALOX15 and ALOX15B was investigated using recombinant enzyme preparations with different substrate PUFAs (Figure 1) (Kutzner et al., 2017).

Omega-6 PUFAs are the most abundant polyenoic fatty acids in mammalian cells and are therefore major lipoxygenase (LOX) substrates. Using AA as substrate, ALOX15 displayed dual reaction specificity forming both 15-HETE and 12-HETE in a 9:1 ratio, confirming original experiments performed with its purified rabbit ortholog (Bryant et al., 1982). In contrast to ALOX15, ALOX15B exhibited singular positional specificity producing only 15-HETE. When LA was used as substrate, 13-HODE was the dominant product for both ALOX15 and ALOX15B. The omega-3 PUFAs, including EPA and DHA, are also present in mammalian cells but at much lower concentrations than omega-6 PUFAs (Calder, 2008). When EPA was employed as substrate, ALOX15 showed a more pronounced dual positional specificity compared to AA producing both 15-HEPA and 12-HEPA in an 8.5:1.5 ratio while ALOX15B exhibited singular positional specificity producing only 15-HEPA. With DHA as substrate, ALOX15-catalyzed oxygenation produced nearly equal amounts of 17-HDHA and 14-HDHA. In contrast, ALOX15B produced 17-HDHA almost

\section{ALOX15}
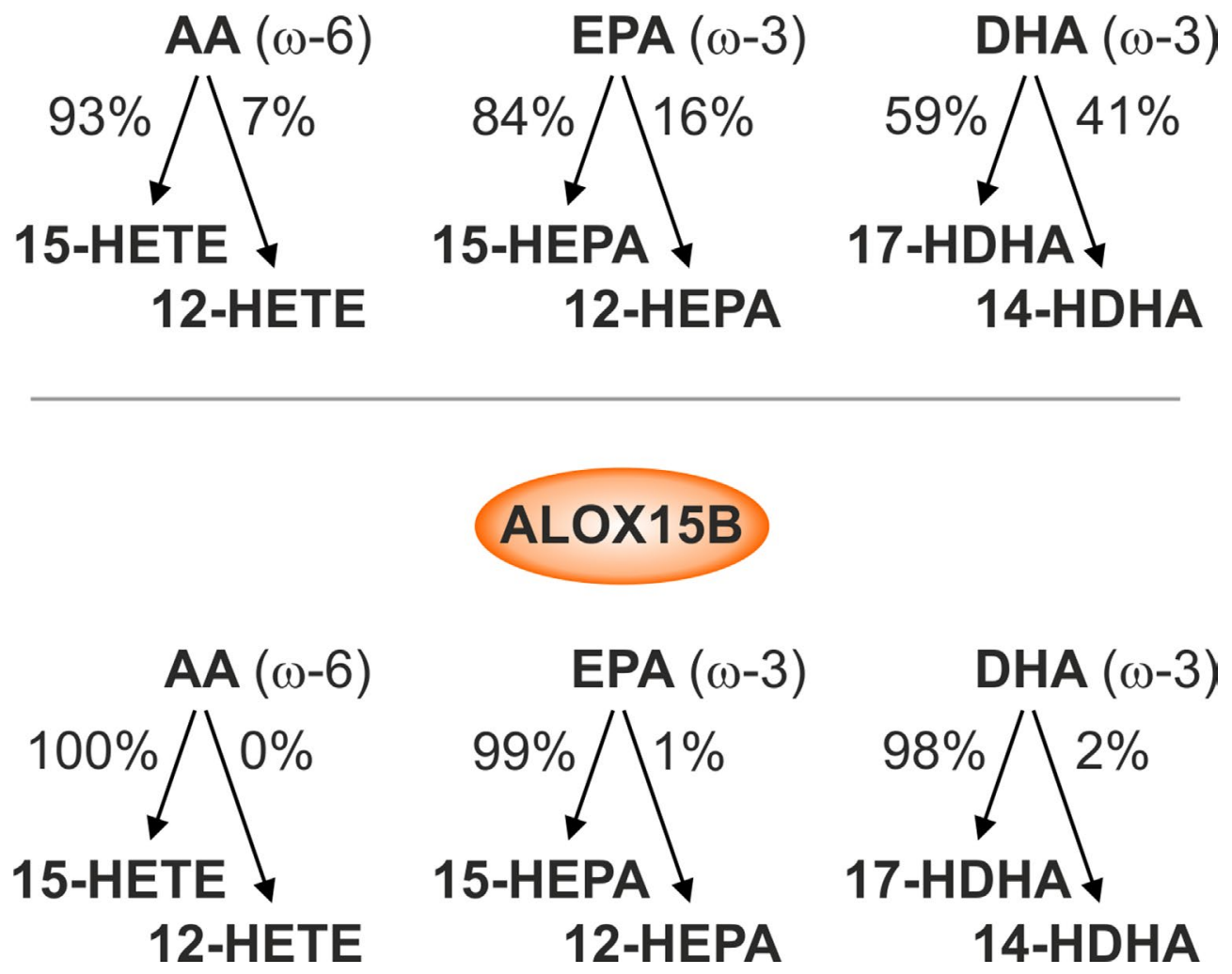

FIGURE 1 | Reaction specificities of human ALOX15 and ALOX15B. Enzymatic activity of recombinant human ALOX15 and ALOX15B with $\omega$ - 6 and $\omega$-3 free polyunsaturated fatty acid (PUFA) substrates. ALOX15 exhibits dual reaction specificity while ALOX15B exhibits singular reaction specificity of arachidonic acid (AA), eicosapentaenoic acid (EPA), and docosahexaenoic acid (DHA) oxygenation. 
exclusively (Kutzner et al., 2017). In vitro, both ALOX15 and ALOX15B produce identical oxygenation products, albeit at different ratios reflecting their singular and dual specificity to free PUFA substrates. The biological implication of these differences is currently unknown; however, the dual reaction specificity unique to ALOX15 that generates 12-HETE, 12-HEPA, and 14-HDHA might be advantageous for SPM biosynthesis in macrophages.

Unlike other mammalian LOX isoforms, both ALOX15 and ALOX15B are unique in their ability to oxygenate esterified PUFAs found in biomembranes, lipoproteins, and cholesteryl esters (CEs) in addition to free fatty acid substrates (Schewe et al., 1975; Belkner et al., 2000; Hammond and O'Donnell, 2012; Hutchins and Murphy, 2012; Bender et al., 2016; Wenzel et al., 2017). In 2007, analysis of ionophore-activated IL-4-treated human monocytes revealed four esterified 15-HETEs, subsequently identified as 3 plasmalogen and 1 acyl phosphatidylethanolamine [18:0p, 18:1p, 16:0p, and 18:0a/15HETE-phosphatidylethanolamine (PE)] (Maskrey et al., 2007). Further analysis revealed that these products were generated by ALOX15 through direct oxidation of the intact phospholipid (PL) and comprised approximately $30 \%$ of the total 15 -HETE generated (O'Donnell and Murphy, 2012). Within the monocyte PL fractions, over $90 \%$ of 15 -HETE was found incorporated in $\mathrm{PE}$, while approximately $1.5 \%$ of the total cellular PE pool was found to contain 15-HETE (O'Donnell and Murphy, 2012). Both human ALOX15B and its murine ortholog Alox15b have also been shown to oxidize esterified PUFAs in solubilized PLs as well as in bilayer PLs encompassed in nanodiscs (Coffa and Brash, 2004; Bender et al., 2016). Following incubation of ALOX15B with PL-esterified-AA containing nanodiscs, 15-HETE but not 12-HETE was formed as product corroborating the in vitro data, which demonstrated the singular positional specificity of ALOX15B with AA substrate (Kutzner et al., 2017). Moreover, when exogenous ALOX15B was added to crude HEK cell lysates, 15-HETE and LA oxygenation product 13-HODE were only detected following treatment with phospholipase $\mathrm{A}_{2}$, likely indicating that the products were generated as the esterified substrate. A key difference between 15-LOX-mediated oxygenated PLs and oxygenated free PUFAs is that oxygenated PLs do not get secreted but remain cell associated, residing within membranes. Although unlikely to mediate high-affinity receptor-ligand interactions, oxidized PLs (oxPLs) likely exert their effects through low-affinity interactions with proteins and by altering membrane electronegativity, which influences protein-membrane interactions (O’Donnell et al., 2019). Emerging evidence indicates that, enzymatically, oxPLs have profound biological activity in macrophages including blocking cell surface and soluble pattern recognition receptors including TLR4, CD14, and LPS-binding protein as well as orchestrating the nonimmunogenic clearance of apoptotic cell (AC)-derived autoantigens and maintaining self-tolerance during inflammation (Uderhardt et al., 2012; Rothe et al., 2015). In non-macrophage immune cells including eosinophils and platelets, ALOX15derived HETE-PLs enhance the ability of phosphatidylserine to interact with multiple clotting factors, increasing the rates of thrombin generation that facilitate hemostasis (Uderhardt et al., 2017; O’Donnell et al., 2019).
Intracellular cholesterol can be esterified with fatty acids to form CEs, which have been shown to be substrates for 15-lipoxygenases (Belkner et al., 1993; Hutchins et al., 2011; Hutchins and Murphy, 2012). Because the oxidation of CEs, which are a major component of low-density lipoproteins (LDLs), is frequently cited in the transformation of macrophages to foam cells during progression of atherosclerotic lesions, the role of 15-lipoxygenases in oxidized $\mathrm{CE}$ formation has been investigated. Human atherosclerotic lesions contain large amounts of oxygenated PUFAs with derivatives of LA being the most predominant (Rapp et al., 1983; Kühn et al., 1992). Of the oxygenated PUFAs in lesions, more than $85 \%$ are localized in CEs (Kühn et al., 1992). Furthermore, it was reported that $23 \%$ of cholesteryl linoleate (18:2-CE), $16 \%$ of cholesteryl arachidonate (20:4-CE), and $12 \%$ of cholesteryl docosahexaenoate (22:6-CE) in human atherosclerotic plaques became oxidized through enzymatic and non-enzymatic processes (Hutchins et al., 2011). To gain insight into the role of 15-lipoxygenase in mediating oxidized CE (oxCE) generation in macrophages, Hutchins et al. measured the oxCEs in wild-type and Alox15-deficient murine resident peritoneal macrophages following incubation with human lipoprotein CEs (Hutchins and Murphy, 2012). Alox15-specific oxidation products of 18:2-CE and 20:4-CE were consistently present in wild-type cells but could not be detected in macrophages lacking Alox15. Further examination of the metabolic fate of Alox15-mediated oxCEs revealed a robust intracellular remodeling pathway whereby hydrolysis of the oxidized fatty acyl chain and subsequent reacylation generated oxidized phosphatidylcholines (PCs) (Hutchins and Murphy, 2012). These results demonstrate that oxCE remodeling contributes to $\mathrm{PC}$ oxidation and suggests that the presence of abundant oxCE may influence overall PC oxidation levels in macrophages. Moreover, oxidation of the acyl chain of the CEs has been suggested to enhance the propensity for hydrolysis as oxidized 18:2-CE was shown to be preferentially hydrolyzed over its non-oxidized counterpart by macrophage $\mathrm{CE}$ hydrolases at both neutral and acidic pH (Belkner et al., 2000). Collectively, these works suggest that by oxidizing intracellular CEs, 15-lipoxygenases may facilitate the hydrolysis and subsequent mobilization of oxidized acyl species for incorporation into various cellular compartments.

Upon cellular stimulation with calcium ionophore, both ALOX15 and ALOX15B increase localization at plasma membrane and at the cytoplasmic side of intracellular membranes (Brinckmann et al., 1998; Bender et al., 2016). Insight into how ALOX15 and ALOX15B undergo localization to biological membranes and selectively oxygenate individual PUFA-PL substrates from the diversified PLs was recently investigated. Using various ALOX15 and ALOX15B-expressing cell types, Wenzel et al. reported that a promiscuous small scaffolding protein, phosphtidylethanolamine-binding protein 1 (PEBP1), forms complexes with ALOX15 and ALOX15B, which can be further increased following stimulation with IL-13 and lipopolysaccharide (LPS), respectively (Wenzel et al., 2017). In the absence of PEBP1, both ALOX15 and ALOX15B exert high enzymatic oxidation of free AA but low activity towards esterified AA-PE. Upon addition of PEBP1, the oxidation of AA-PE by both LOX isoforms was 
markedly increased. Mechanistic insight showed that PEBP1 contains multiple sites for binding free AA and through binding reduces AA levels accessible for oxygenation. By "depleting" endogenous AA and complexing with ALOX15 or ALOX15B, PEBP1 redirects AA-PE as enzymatic substrate for 15-LOXs. Whether PEBP1 binds other PUFAs in addition to AA to facilitate oxygenation of the corresponding PUFA-PLs was not reported.

\section{REGULATION OF ALOX15 AND ALOX15B EXPRESSION}

In contrast to ALOX15, which is absent in unstimulated human monocyte-derived macrophages, ALOX15B is constitutively expressed at both messenger RNA (mRNA) and protein levels, and its expression can be further enhanced by bacterial-derived pro-inflammatory stimulus LPS and immune regulatory T-helper type 2 cytokines IL-4 and IL-13 (Figure 2) (Wuest et al., 2012; Snodgrass et al., 2018).

Gene and protein expression levels as well as enzymatic activity of ALOX15B were also increased in primary human macrophages incubated under hypoxia and following treatment with dimethyloxalylglycine, which mimics low oxygen tension by stabilizing the transcription factor hypoxia-inducible factor 1a (HIF-1a) (Rydberg et al., 2004; Hultén et al., 2010). Neither dimethyloxalylglycine nor hypoxia increased expression of ALOX15. Furthermore, knockdown of HIF-1a in hypoxic macrophages reduced production of the 15-lipoxygenasemediated AA metabolite 15-HETE, further implicating a role for HIF-1 $\alpha$ in the hypoxic induction of ALOX15B. More detailed studies investigating the regulation of $A L O X 15 B$ gene expression were performed in normal human prostate (HNP) epithelial cells (Tang et al., 2004; Bhatia et al., 2005). Analysis revealed that the ALOX15B promoter is not TATA box-enriched, consistent with the constitutive expression in vivo, and contains several Sp1 sites critical for regulating gene expression. Subsequent experiments identified Sp1 and Sp3 as major GC-binding trans factors regulating $A L O X 15 B$ gene expression. Sp1 activated while Sp3 inhibited $A L O X 15 B$ promoter activity, and endogenous $A L O X 15 B$ expression in HNP cells established Sp1 and Sp3 as biologically relevant and essential regulators of the ALOX15B gene (Tang et al., 2004).

Several splice variants of ALOX15B were identified in HNP epithelial cells and prostate cancer (PCa) cells (Bhatia et al., 2003; Bhatia et al., 2005; Tang et al., 2007). All identified variants had spliced out key segments of the protein important for its catalytic activity, leaving the enzyme with little to no AA-metabolizing activity. Unlike the full-length protein, the alternatively spliced isoforms were also found to be largely excluded from the nucleus.
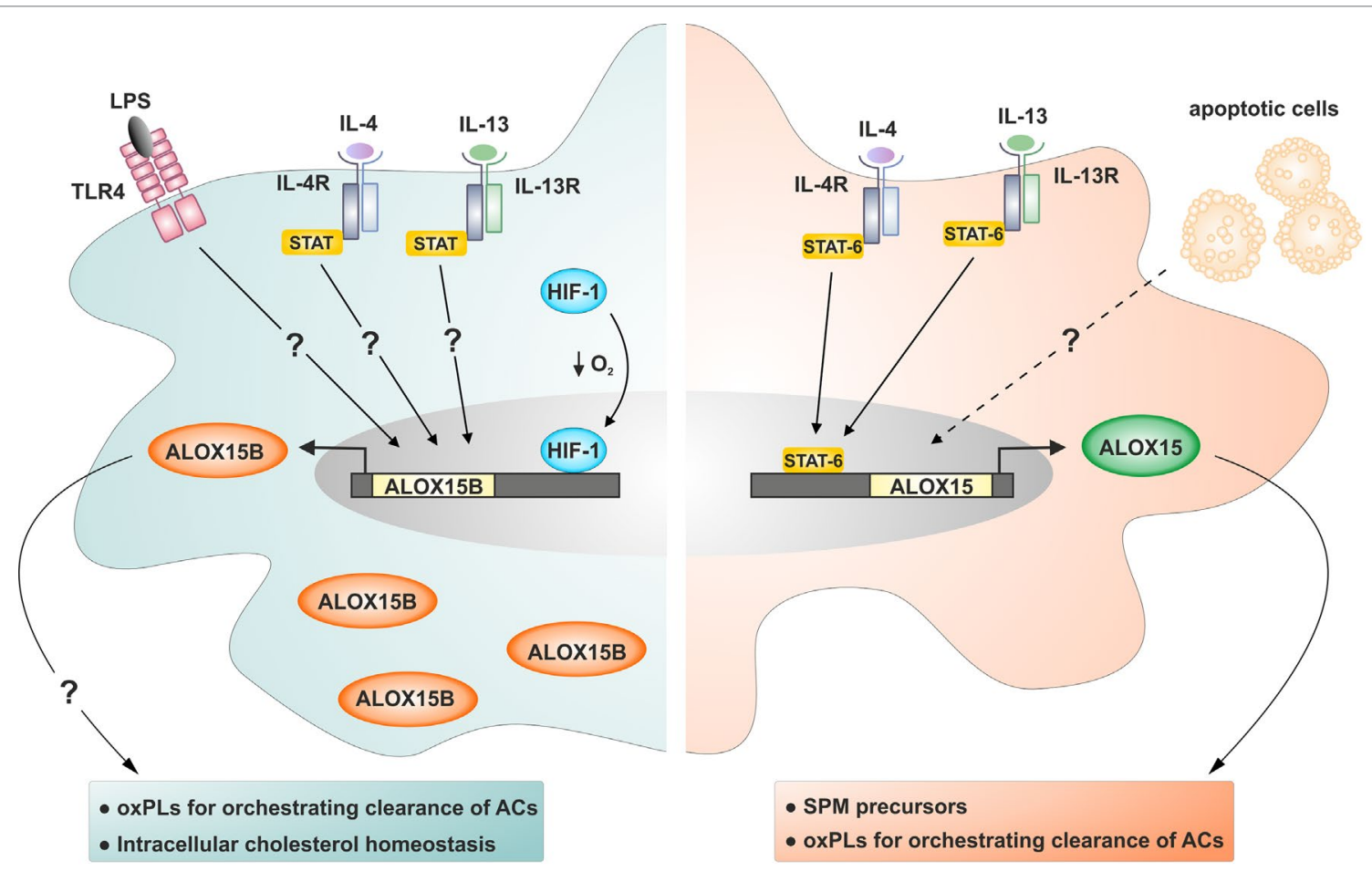

FIGURE 2 | Regulation of ALOX15B and ALOX15 expression in human macrophages. ALOX15B protein is constitutively present in human monocyte-derived macrophages and can be further induced by stimulation with bacterial-derived pro-inflammatory stimuli LPS, immune regulatory T-helper type 2 cytokines IL-4 and IL-13, and HIF-1 following reduced oxygen tension. ALOX15 is absent in human monocyte-derived macrophages and requires stimulation by IL-4 or IL-13 for protein induction. Macrophages exposed to apoptotic cells (ACs) and IL-4 or IL-13 display pro-resolving properties exemplified by heightened ALOX15 expression. Question marks indicate uncertain signaling pathways. 
In gain-of-function experiments, splice variant-expressing cells displayed similar biological activities to the full-length protein with respect to inhibiting cell-cycle progression and tumor development and inducing cell senescence. These results suggest that the tumor-suppressive functions of ALOX15B and its splice variants do not necessarily depend on AA-metabolizing activity and nuclear localization and support a biological function for ALOX15B independent of PUFA metabolism.

In human monocyte-derived macrophages, ALOX15 expression is strictly cytokine-dependent and is strongly up-regulated following stimulation with IL-4 or IL-13 (Conrad et al., 1992; Czimmerer et al., 2012; Gundra et al., 2014; Ackermann et al., 2017). Stimulation with other cytokines including IL-1 $\beta$, IL-6, TNF- $\alpha$, TGF- $\beta$, and IFN $\gamma$, by agonists of TLR3, TLR4, TLR7, TLR8, TLR9, as well as hypoxia, did not induce expression of ALOX15 mRNA or protein (Rapp et al., 1983; Belkner et al., 1993; Wuest et al., 2012). Maximal protein induction is reached after incubation periods longer than $48 \mathrm{~h}$, suggesting that $A L O X 15$ does not belong to the immediate early genes of the IL- 4 response (Wuest et al., 2012; Werz et al., 2018). In vitro experiments posit that activation of the transcription factor signal transducer and activator of transcription (STAT)- 6 by IL- 4 or IL-13 is indispensable for induction of ALOX15 gene transcription in monocyte-derived macrophages (Heydeck et al., 1998; Han et al., 2014). Although both IL-4 and IL-13 signal through receptor systems containing the IL-4Ra component to induce ALOX15 in a STAT6-dependent manner, their intracellular signaling mechanisms are distinct. IL-4 utilizes the IL-4R $\alpha / J a k 1$ cascade to activate STAT6 and STAT3 whereas IL-13 utilizes both IL-4Ra/Jak2 and IL-13Ra1/ Tyk2 to activate STAT6, STAT3, and STAT1 (Bhattacharjee et al., 2013). Histone modifications and chromatin remodeling also play critical roles in $A L O X 15$ gene expression. In human lung epithelial carcinoma A549 cells, IL-4 activated the histone acetyltransferase activity of the CAMP response element binding protein (CREB)-binding protein (CBP)/p300, which is responsible for acetylation of nuclear histones and STAT6 (Shankaranarayanan et al., 2001). Inhibition of its acetyltransferase activity abrogated acetylation of both histones and STAT6 and strongly suppressed transcriptional activation of $A L O X 15$. In the Hodgkin lymphoma cell line L1236, which constitutively express ALOX15, abolishing histone methyltransferase SMYD3 reduced ALOX15 expression by reducing di-/trimethylation of histone 3 lysine 4 (H3-K4), attenuated occupancy of STAT6, and diminishing histone H3 acetylation at the ALOX15 promoter. In contrast to L1236 cells, inhibiting JmjC-domain-containing H3-K4 tri-demethylase lysine demethylase 5C (KDM5C) in the Hodgkin lymphoma cell line L428, which does not express $A L O X 15$, upregulated $A L O X 15$ expression through inducing $\mathrm{H} 3-\mathrm{K} 4$ trimethylation, histone acetylation, and STAT6 recruitment at the ALOX15 promoter (Liu et al., 2012). In A549 cells but not human peripheral monocytes, IL-4 stimulation induced H3K27me2/3-specific demethylase (UTX)-mediated H3K27me3 demethylation at the ALOX15 promoter, triggering mRNA transcription and protein expression (Han et al., 2014). Further investigations showed that IL-13-mediated ALOX15 gene expression in human primary monocytes involves ERK1/2dependent signaling cascades that induce transcription factor early growth response-1 (Egr-1) nuclear accumulation and CREB serine phosphorylation and subsequent DNA binding to their cognate sequences within the ALOX15 promoter (Bhattacharjee et al., 2010). In primary human monocyte-derived macrophages, IL-4induced $A L O X 15$ expression was attenuated by AMP-activated protein kinase (AMPK) activation. AMPK activation inhibited IL-4-evoked STAT3 but not STAT6 activation. In addition, AMPK activation prevented IL-4-induced association of STAT6 and Lys-9 acetylation of histone $\mathrm{H} 3$ at the ALOX15 gene promoter (Namgaladze et al., 2015).

Currently, the only known mode of ALOX15 induction in monocytes and macrophages is through stimulation with classical Th2 cytokines IL-4 or IL-13 (Kuhn et al., 2016). However, the discrepancy between concentrations of IL- 4 and IL-13 required to induce ALOX15 in cell culture models (typically $1-50 \mathrm{ng} / \mathrm{ml}$ ) with those measured in human plasma (low $\mathrm{pg} / \mathrm{ml}$ range) questions the biological relevance of IL-4- and IL-13-induced upregulation of ALOX15 expression (Kuhn et al., 2016). Considerations about the dispensable nature of IL-4- and IL-13-induced ALOX15 expression in vivo stem from early investigations comparing ALOX15 expression in human monocyte-derived macrophages matured in vitro with in vivo-matured mouse peritoneal macrophages. In 1996, it was shown that mouse peritoneal macrophages possess considerable Alox15 activity and protein in the absence of exogenous IL-4 and when harvested from IL-4-deficient mice (Cornicelli et al., 1996). However, the subsequent discovery that IL-13, which uses shared receptor subunits to activate common pathways as IL-4, mimics the effect of IL-4 on ALOX15 expression in monocytes and macrophages (Nassar et al., 1994; Heydeck et al., 1998; Chaitidis et al., 2005) likely account for the lack of reduced Alox15 expression in macrophages isolated from IL-4-deficient mice. Follow-up experiments investigating the dispensable nature of lymphocyte-derived IL-4 and IL-13 in Alox15 expression in vivo assessed Alox15 levels in peritoneal macrophages harvested from recombinase activator gene (RAG)-2 knockout mice deficient in Th2 cytokine-producing mature lymphocytes (Sendobry et al., 1998). In contrast to the researchers' expectations, Alox15 levels in macrophages isolated from knockout mice were not decreased. However, in the 20 years since this publication, many additional cell types in addition to mature $\mathrm{T}$ lymphocytes have been shown to produce IL-4 or IL-13 including NK T cells, basophils, mast cells, eosinophils, group 2 innate lymphoid cells (ILC2 cells), and multipotent progenitors type 2 cells (Molofsky et al., 2013; Paul, 2015; Yamanishi and Karasuyama, 2016). More recent studies have shown that the sensing of IL-4 or IL-13 together with the recognition and integration of ACs through members of the TAM (Tyro3, Axl, and Mer) receptors, which are expressed on professional phagocytes and contribute to AC clearance, enhances anti-inflammatory and tissue repair gene expression including Alox15 (Bosurgi et al., 2017). ACs are generated not only during inflammation but also under normal physiological conditions (Poon et al., 2014), resulting in more than $10^{11} \mathrm{ACs}$ cleared in the normal adult mammal on a daily basis (Henson, 2017). Considering the ubiquity of ACs in vivo and their demonstrated ability to enhance IL-4- and IL-13induced Alox15 expression in murine macrophages, investigating how macrophages integrate concurrent recognition of biological factors and cytokine receptor signals may reveal novel mechanisms coordinating ALOX15 expression in vivo. 


\section{BIOLOGICAL FUNCTION OF ALOX15 AND ALOX15B IN HUMAN MACROPHAGES}

\section{Production of SPMs}

Initiation and resolution of inflammation are finely regulated by chemical messengers including lipid mediators (Spite et al., 2014). Whereas AA-derived prostaglandins and leukotrienes formed by the cyclooxygenase and arachidonate 5-lipoxygenase (ALOX5) pathways initiate acute inflammation, the process of terminating inflammation and promoting resolution are coordinated by a group of temporally produced lipids called SPMs. The SPM superfamily consist of LXs synthesized from AA, E-series Rvs from EPA, and DHA-derived D-series Rvs, protectins, and maresins (Spite et al., 2014). While enzyme activity assays show both ALOX15 and ALOX15B synthesize SPM precursor lipids including 15-HETE used for LX synthesis and 17-HDHA used for the synthesis of Rvs (Kutzner et al., 2017), biological data in macrophages posit that only ALOX15 contribute to this process. Stimulation of ALOX15B-expressing human monocytederived macrophages with IL-4 increased protein expression of ALOX15 and cellular levels of 15-LOX-synthesized SPM precursor 15-HETE and monohydroxy LA metabolite 13-HODE (Snodgrass et al., 2018). In these cells, only knockdown of ALOX15, but not ALOX15B, decreased the lipid metabolites to basal levels, suggesting that only ALOX15 contributes to SPM precursor generation. Moreover, LPS- and interferon- $\gamma$ polarized classically activated macrophages expressing ALOX5, 5-lipoxygenase-activating protein (FLAP), but not ALOX15 challenged with pathogenic Escherichia coli produced large amounts of prostaglandin $\mathrm{E}_{2}$ and leukotriene $\mathrm{B}_{4}$ but no SPMs or 15-LOX-derived SPM precursors (Werz et al., 2018; Werner et al., 2019). In IL-4-polarized alternatively activated macrophages, which strongly express ALOX15 and contain low levels of FLAP, incubation with E. coli produced 15-LOX-derived SPM precursors (15-HETE, 17-HDHA, and 15-HEPA) and SPMs (RvD2, RvD5, LXs, and maresin). Although ALOX15B protein is constitutively expressed in human macrophages (Wuest et al., 2012; Snodgrass et al., 2018), only ALOX15 protein appears to contribute to the production of SPMs and its precursors.

\section{ACs and Efferocytosis}

ALOX15 is highly expressed in IL-4- and IL-13-induced alternatively activated macrophages in vitro and in macrophages participating in the resolution of inflammation in vivo (SchifZuck et al., 2011; Czimmerer et al., 2012; Uderhardt et al., 2012; Gundra et al., 2014; Ackermann et al., 2017; Snodgrass et al., 2018; Werz et al., 2018; Yang et al., 2019). Transcriptomic analysis of resolution-phase macrophages showed them to be enriched in genes involved in antigen processing and presentation as well as genes involved in dampening leukocyte trafficking, wound repair, and efferocytosis (Stables et al., 2011). Subsequent analysis of Alox15-expressing resolution-phase macrophages isolated from models of resolving peritonitis and acute $\mathrm{N}$-acetylp-aminophenol-induced liver injury indicated a heightened efferocytic capacity (Schif-Zuck et al., 2011; Uderhardt et al., 2012;
Yang et al., 2019). However, rather than facilitating the uptake of ACs, Alox15 expression appears to be a consequence of AC engulfment, as post-efferocytotic "satiated" Alox15-expressing macrophages were shown to display reduced responsiveness to TLR ligands and low phagocytic potential, and were prone to migrate to lymphoid organs (Schif-Zuck et al., 2011). An independent study of peritonitis also excluded an intrinsic role for Alox15 during the ingestion of ACs as resolution-phase macrophages from both wild-type and Alox15 knockout mice ingested equally high amounts of ACs (Uderhardt et al., 2012). Although likely not required for the direct uptake of ACs, the enzyme appears to be a crucial factor orchestrating clearance of ACs as resident peritoneal macrophages utilize Alox15 to generate specific oxidation products of $\mathrm{PE}$, which block uptake of ACs from recruited inflammatory monocytes. This selective phagocytosis prevents efferocytosis by inflammatory monocytes and subsequent antigen presentation of AC-derived antigens, thus maintaining self-tolerance.

Recent studies from animal models suggest the reprogramming of AC-engulfing macrophages to an Alox15-expressing, proresolving, and tissue repair phenotype that involves Axl and Mer tyrosine kinase (MerTK) TAM receptors (Bosurgi et al., 2017; Lumbroso et al., 2018). Mice infected with the helminth Nippostrongylus brasiliensis develop substantial lung tissue damage followed by a rapid IL- 4 and IL-13 response, which is critical for resolution of inflammation and tissue repair. Lungresident macrophages isolated 7 days post $N$. brasiliensisinfection showed increased expression of Alox15 as well as anti-inflammatory and tissue repair genes that were substantially reduced in mice with macrophage-specific ablation of the $\mathrm{AC}$ sensors Axl and MerTK (Bosurgi et al., 2017). The expression of IL-4-induced genes that were independent of AC sensing was not impaired in macrophages lacking Axl and MerTK. To gain further insight into macrophage reprogramming by TAM receptor signaling, Lumbroso et al. utilized macrophages deficient in the bridging molecule Pros1, which binds PS on ACs to allow TAM receptor engagement, in a zymosan-induced peritonitis mouse model (Lumbroso et al., 2018). Pros1-deficient macrophages collected from peritoneal exudates $66 \mathrm{~h}$ post zymosan treatment engulfed fewer apoptotic polymorphonuclear cells (PMNs) compared to control cells. Moreover, Pros1-deficient peritoneal macrophages displayed reduced reprogramming following apoptotic neutrophil engulfment as indicated by increased secretion of pro-inflammatory mediators and decreased levels of anti-inflammatory cytokines following exposure to LPS. Pros1deficient peritoneal macrophages also expressed reduced levels of Alox 15 protein and produced 25\% less RvD1 compared to control cells. Collectively, these studies indicate the significance of the coordination of IL-4 or IL-13 with AC sensing in macrophages in inducing ALOX15 and the anti-inflammatory and tissue repair phenotype. Although the molecular signaling mechanisms that integrate IL-4, IL-13, and AC sensing to reprogram macrophages remain unknown, it is interesting to consider how constitutively expressed ALOX15B might play a role in this process and whether it displays any intrinsic or coordinating role in efferocytosis. 


\section{Atherosclerosis}

The role of ALOX15 and ALOX15B in atherogenesis is complex due in part to the fact that atherosclerosis is a multifactorial disease entailing a complex interplay of modified lipoproteins, monocyte-derived macrophages, T cells, and the arterial wall. Since macrophages play a central role in atherogenesis, understanding the role of macrophage-specific 15-LOXs in lipid handling and foam cell formation has been of great interest. Oxidative modification of LDL particles in the artery wall leads to the formation of atherogenic oxidized LDL (oxLDL). oxLDL can be recognized and taken up by macrophages where the accumulation of cholesterol converts them into foam cells, which initiate the development of atherosclerotic lesions. Although the precise mechanisms that generate oxLDL in vivo are still only partially understood, both ALOX15 and ALOX15B may contribute to the formation of atherogenic oxLDL. Reports have shown that rabbit, mouse, porcine, and human ALOX15 directly oxidizes LDL particles and contributes to foam cell formation (Belkner et al., 1993; Kühn et al., 1994; Kühn and Chan, 1997; Belkner et al., 1998; Heydeck et al., 2001). Although these results support the notion that ALOX15 can directly contribute to atherosclerosis via LDL oxidation, subsequent studies performed in transgenic animal models reported both pro-atherogenic and anti-atherogenic roles for ALOX15 (Shen et al., 1996; Cyrus et al., 1999; Harats et al., 2000; Merched et al., 2008). Early studies that analyzed human atherosclerotic plaques reported increased levels of enzymatically derived 13(S)-HODE compared to the nonenzymatically-derived 13(R)-HODE, suggesting the presence of ALOX15 and its contribution to the formation of atherosclerotic lesions (Kühn et al., 1994; Folcik et al., 1995; Kühn et al., 1997). However, with the discovery of a second 15-lipoxygenase, ALOX15B, a more thorough analysis and comparison of the role of the 15-LOXs in atherosclerosis became warranted. It is now well established that human atherosclerotic plaques contain large amounts of 15-lipoxygenase-derived lipid metabolites of AA, EPA, and DHA; however, the respective contribution of ALOX15 and ALOX15B is unclear (Fredman et al., 2016). Using a collection of human carotid plaque tissue, Gertow et al. reported that $A L O X 15 B$ was highly expressed in the carotid lesions while immunohistochemical analysis showed abundant ALOX15B protein expression in macrophagerich lesion areas (Gertow et al., 2011). In contrast to ALOX15B, ALOX15 expression was not detected in carotid lesions. A second study using human carotid plaques from patients with high-grade symptomatic carotid artery stenosis also reported high ALOX15B but not ALOX15 protein levels in carotid lesions (Hultén et al., 2010). Immunohistochemistry analysis showed that ALOX15B expression in carotid endarterectomies correlated with the expression of HIF- $1 \alpha$. Moreover, $A L O X 15 B$ gene expression in CD14+ macrophages isolated from the human carotid endarterectomies was 500 times higher than ALOX15. To investigate the role of ALOX15B in promoting the development of atherosclerosis in vivo, researchers used lentiviral shRNA silencing and bone marrow transplantation to knock down Alox15b in LDL-receptor-deficient mice (Magnusson et al., 2012). Immunohistochemical analysis indicated mice that received Alox15b knockdown bone marrow had reduced atherosclerotic lesions in both whole aorta and aortic root compared to nonsilencing control mice. In summary, although in vitro data support a role for ALOX15 and ALOX15B in atherogenesis, data from in vivo animal experiments and from human carotid plaque tissue implicate a role for ALOX15B rather than ALOX15.

\section{Cellular Lipid Homeostasis}

Various studies have reported roles for the 15-LOX enzymes in regulating macrophage lipid homeostasis (Belkner et al., 2005; Weibel et al., 2009; Rong et al., 2012; Snodgrass et al., 2018). Initial experiments found that overexpressing porcine Alox15 in J774 murine macrophages protected cells from intracellular lipid deposition following incubation with acetylated LDL (Belkner et al., 2005). Further analysis discovered that porcine Alox15overexpressing macrophages accumulated less intracellular CEs due to increased CE catabolism. Administration of Alox15mediated metabolites of AA and LA, 12-HETE and 13-HODE, failed to reduce intracellular CE accumulation in control cells, suggesting a mechanism mediated via metabolites of free and/ or esterified hydroperoxy lipids formed from Alox15-catalyzed fatty acid oxygenation. In a similar experiment, overexpression of human ALOX15 in RAW 267.4 murine macrophages led to increased intracellular CE hydrolysis, elevated ABCA1 protein levels, and enhanced cholesterol efflux, suggesting that the CEs produced in ALOX15-expressing cells are readily mobilized for ABCA1-mediated cholesterol efflux (Weibel et al., 2009). The increased intracellular CE hydrolysis observed in ALOX15expressing macrophages was not attributable to increased neutral cholesterol ester hydrolase activity, suggesting that the CEs in ALOX15-expressing cells are a better substrate for neutral cholesterol ester hydrolase compared to CEs in control cells. Although consistent with previous experiments showing that ALOX15 oxygenates intracellular CE, levels of oxCE in the ALOX15-overexpressing and control cells were not measured in these experiments. In an animal model of atherosclerosis, Alox15/Ldl receptor double knockout mice fed a PUFAenriched diet had reduced plasma cholesterol and triglyceride levels, liver lipid levels, and aortic atherosclerosis compared to Ldl receptor knockout mice (Rong et al., 2012). Hepatic gene expression revealed that double knockout mice had reduced levels of fatty acid and triglyceride synthesis-related genes sterol regulatory element binding protein-1c (Srebp-1c), fatty acid synthase (Fas), acetyl-CoA carboxylase-1 (Acc-1), stearoyl-CoA desaturase-1 $(S c d-1)$, as well as cholesterol synthetic regulatory genes Srebp-2 and 3-hydroxy-3-methylglutaryl-CoA ( Hmg-CoA) synthase and reductase compared with single knockout controls. Considering that ALOX15 is expressed in macrophages, not hepatocytes, the authors concluded that macrophage ALOX15 expression altered secretory products that affected hepatic lipid synthesis. With respect to lipid and sterol homeostasis in macrophages, we recently reported that suppressing ALOX15B and, to a lesser extent, ALOX15 in human primary monocytederived macrophages impaired SREBP-2 signaling by inhibiting SREBP-2 processing into mature transcription factor and reduced SREBP-2 binding to sterol regulatory elements and subsequent target gene expression (Snodgrass et al., 2018). In IL-4 stimulated human macrophages, which express both ALOX15 and ALOX15B proteins, silencing ALOX15B but not ALOX15 
reduced cellular cholesterol and the cholesterol intermediates desmosterol, lanosterol, 24,25-dihydrolanosterol, and lathosterol as well as oxysterol 27-hydroxycholesterol. In addition to reduced expression of SREBP-2 target genes, knockdown of ALOX15B increased expression of liver $\mathrm{X}$ receptor (LXR) target genes ABCG1, ABCA1, and MYLIP following stimulation with IL-4. In agreement with previous reports, attempts to rescue alterations in SREBP-2 target gene expression in 15-LOX-suppressed macrophages with exogenous AA-, LA-, or DHA-derived 15-LOX metabolites failed. Collectively, our results are in agreement with previous reports suggesting a role for 15-lipoxygenases in regulating lipid and sterol homeostasis in macrophages through a currently undefined mechanism.

\section{Potential Functions of Macrophage ALOX15B}

In contrast to macrophage ALOX15, which is strongly implicated in inflammation resolution and orchestrating efferocytosis, no compelling data currently exist to suggest a role for ALOX15B in either of these processes. Whereas most of the in vivo biological functions of ALOX15 have been elucidated using knockout mice, Alox15b-deficient mice are not commercially available. Likewise, many potent small-molecule inhibitors targeting ALOX15 have been reported (Rai et al., 2013; Sadeghian and Jabbari, 2016) and are commercially available, including PD146176 and ML351; however, inhibitors exhibiting potent and selective inhibition of ALOX15B do not currently exist. Therefore, the limited collective knowledge of the biological functions of ALOX15B is derived primarily from in vitro experimentation through manipulating its expression in various cell culture models. While the function of ALOX15B in macrophages is rather unclear, its role in non-myeloid cells including human epithelial cells of the prostate, skin, esophagus, and cornea has been more thoroughly investigated (Tang et al., 2002; Schweiger et al., 2007; Tang et al., 2007; Suraneni et al., 2014). In epithelial cells, ALOX15B functions as a regulator of cell senescence (Schweiger et al., 2007; Tang et al., 2007; Suraneni et al., 2010). Research in human prostate cells has shown that ALOX15B expression is down-regulated or lost in the precursor lesion HGPIN (high-grade prostate intraepithelial neoplasia) as well as in $>70 \%$ of prostate cancers (Shappell et al., 1999; Jack et al., 2000; Tang et al., 2002; Tang et al., 2007; Suraneni et al., 2014). In nearly all immortalized prostate epithelial and PCa cell lines, the expression of ALOX15B is undetectable. Furthermore, restoring its expression in $\mathrm{PCa}$ cells inhibits proliferation, induces senescence-like phenotypes, and abrogates tumor regeneration in xenograft models (Suraneni et al., 2014). Although mechanisms by which ALOX15B functions as a negative cell cycle regulator are not

\section{REFERENCES}

Ackermann, J. A., Hofheinz, K., Zaiss, M. M., and Krönke, G. (2017). The double-edged role of 12/15-lipoxygenase during inflammation and immunity. Biochim. Biophys. Acta Mol. Cell. Biol. Lipids 1862, 371-381. doi: 10.1016/j.bbalip.2016.07.014

Adel, S., Karst, F., González-Lafont, À, Pekárová, M., Saura, P., Masgrau, L., et al. (2016). Evolutionary alteration of ALOX15 specificity optimizes the biosynthesis of antiinflammatory and proresolving lipoxins. Proc. Natl. Acad. Sci. U.S.A. 113, E4266-E4275. doi: 10.1073/pnas.1604029113 well characterized, it likely possesses multiple biological functions as gain-of-function experiments using ALOX15B splice variants lacking AA-metabolizing activity produced identical biological activities compared with the full length protein. These activities include the inhibition of cell-cycle progression and proliferation, induction of a senescence-like phenotype, and inhibition of tumor development in vivo. To gain a better understanding of how ALOX15B and its splice variants contribute to cellular homeostasis in health and disease, additional research is needed to elucidate the cellular functions of ALOX15B.

\section{CONCLUSION}

Since the discovery of ALOX15 in 1975 (Schewe et al., 1975), an understanding of its function and physiological significance has been extensively pursued. It is now well established that ALOX15 is an IL-4- and IL-13-inducible enzyme in human monocytederived macrophages that catalyzes the oxygenation of free and esterified PUFAs. Through its lipid-metabolizing activity, macrophage ALOX15 plays a central role in generating SPMs to resolve acute inflammation and to produce oxPLs to orchestrate the nonimmunogenic removal of ACs. In 1997, a second AA 15-lipoxygenating enzyme was discovered in human skin and, based on LOX nomenclature, was given the name ALOX15B (Brash et al., 1997). Since then, comparative analyses of ALOX15 and ALOX15B have shown that the former enzyme exhibits dual reaction specificity with several polyenoic fatty acids while the latter exhibits singular reaction specificity. In contrast to ALOX15, which is absent in unstimulated human monocytederived macrophages, ALOX15B is constitutively expressed but does not appear to play a major role in inflammation resolution or orchestrating efferocytosis. Although several correlations between ALOX15B expression and biological activities in nonmacrophage cells have been reported, the function of ALOX15B in human macrophages remains elusive.

\section{AUTHOR CONTRIBUTIONS}

This manuscript was conceptualized by RS and BB, written by RS, and edited by RS and BB.

\section{FUNDING}

This work was supported by the Deutsche Forschungsgemeinschaft (SFB 1039, TPB04 and GRK 2336, TP06).

Belkner, J., Wiesner, R., Rathman, J., Barnett, J., Sigal, E., and Kühn, H. (1993) Oxygenation of lipoproteins by mammalian lipoxygenases. Eur. J. Biochem. 213, 251-261. doi: 10.1111/j.1432-1033.1993.tb17755.x

Belkner, J., Stender, H., and Kühn, H. (1998). The rabbit 15-lipoxygenase preferentially oxygenates LDL cholesterol esters, and this reaction does not require vitamin E. J. Biol. Chem. 273, 23225-23232. doi: 10.1074/ jbc.273.36.23225

Belkner, J., Stender, H., Holzhütter, H. G., Holm, C., and Kühn, H. (2000). Macrophage cholesteryl ester hydrolases and hormone-sensitive lipase prefer 
specifically oxidized cholesteryl esters as substrates over their non-oxidized counterparts. Biochem. J. 352 Pt 1, 125-133. doi: 10.1042/bj3520125

Belkner, J., Chaitidis, P., Stender, H., Gerth, C., Kuban, R. J., Yoshimoto, T., et al. (2005). Expression of 12/15-lipoxygenase attenuates intracellular lipid deposition during in vitro foam cell formation. Arterioscler. Thromb. Vasc. Biol. 25, 797-802. doi: 10.1161/01.ATV.0000157580.26858.2d

Bender, G., Schexnaydre, E. E., Murphy, R. C., Uhlson, C., and Newcomer, M. E. (2016). Membrane-dependent activities of human 15-LOX-2 and its murine counterpart: implications for murine models of atherosclerosis. J. Biol. Chem. 291, 19413-19424. doi: 10.1074/jbc.M116.741454

Bhatia, B., Maldonado, C. J., Tang, S., Chandra, D., Klein, R. D., Chopra, D., et al. (2003). Subcellular localization and tumor-suppressive functions of 15-lipoxygenase 2 (15-LOX2) and its splice variants. J. Biol. Chem. 278, 2509125100. doi: 10.1074/jbc.M301920200

Bhatia, B., Tang, S., Yang, P., Doll, A., Aumüeller, G., Newman, R. A., et al. (2005). Cell-autonomous induction of functional tumor suppressor 15-lipoxygenase 2 (15-LOX2) contributes to replicative senescence of human prostate progenitor cells. Oncogene 24, 3583-3595. doi: 10.1038/sj.onc. 1208406

Bhattacharjee, A., Mulya, A., Pal, S., Roy, B., Feldman, G. M., and Cathcart, M. K. (2010). Monocyte 15-lipoxygenase gene expression requires ERK1/2 MAPK activity. J. Immunol. 185, 5211-5224. doi: 10.4049/jimmunol.1000514

Bhattacharjee, A., Shukla, M., Yakubenko, V. P., Mulya, A., Kundu, S., and Cathcart, M. K. (2013). IL-4 and IL-13 employ discrete signaling pathways for target gene expression in alternatively activated monocytes/macrophages. Free Radic. Biol. Med. 54, 1-16. doi: 10.1016/j.freeradbiomed.2012.10.553

Bosurgi, L., Cao, Y. G., Cabeza-Cabrerizo, M., Tucci, A., Hughes, L. D., Kong, Y., et al. (2017). Macrophage function in tissue repair and remodeling requires IL-4 or IL-13 with apoptotic cells. Science 356, 1072-1076. doi: 10.1126/ science.aai8132

Brash, A. R., Boeglin, W. E., and Chang, M. S. (1997). Discovery of a second 15S-lipoxygenase in humans. Proc. Natl. Acad. Sci. 94, 6148-6152. doi: 10.1073/ pnas.94.12.6148

Brinckmann, R., Schnurr, K., Heydeck, D., Rosenbach, T., Kolde, G., and Kühn, H. (1998). Membrane translocation of 15-lipoxygenase in hematopoietic cells is calcium-dependent and activates the oxygenase activity of the enzyme. Blood 91, 64-74.

Bryant, R. W., Bailey, J. M., Schewe, T., and Rapoport, S. M. (1982). Positional specificity of a reticulocyte lipoxygenase. conversion of arachidonic acid to 15-S-hydroperoxy-eicosatetraenoic acid. J. Biol. Chem. 257, 6050-6055.

Buckley, C. D., Gilroy, D. W., and Serhan, C. N. (2014). Proresolving lipid mediators and mechanisms in the resolution of acute inflammation. Immunity 40, 315-327. doi: 10.1016/j.immuni.2014.02.009

Calder, P. C. (2008). The relationship between the fatty acid composition of immune cells and their function. Prostaglandins Leukot. Essent. Fatty Acids 79, 101-108. doi: 10.1016/j.plefa.2008.09.016

Chaitidis, P., O’Donnell, V., Kuban, R. J., Bermudez-Fajardo, A., Ungethuem, U., and Kühn, H. (2005). Gene expression alterations of human peripheral blood monocytes induced by medium-term treatment with the TH2cytokines interleukin-4 and -13. Cytokine 30, 366-377. doi: 10.1016/j. cyto.2005.02.004

Coffa, G., and Brash, A. R. (2004). A single active site residue directs oxygenation stereospecificity in lipoxygenases: stereocontrol is linked to the position of oxygenation. Proc. Natl. Acad. Sci. 101, 15579-15584. doi: 10.1073/ pnas.0406727101

Conrad, D. J., Kuhn, H., Mulkins, M., Highland, E., and Sigal, E. (1992). Specific inflammatory cytokines regulate the expression of human monocyte 15-lipoxygenase. Proc. Natl. Acad. Sci. U.S.A. 89, 217-221. doi: 10.1073/pnas.89.1.217

Cornicelli, J. A., Welch, K., Auerbach, B., Feinmark, S. J., and Daugherty, A. (1996). Mouse peritoneal macrophages contain abundant omega-6 lipoxygenase activity that is independent of interleukin-4. Arterioscler. Thromb. Vasc. Biol. 16, 1488-1494. doi: 10.1161/01.ATV.16.12.1488

Cyrus, T., Witztum, J. L., Rader, D. J., Tangirala, R., Fazio, S., Linton, M. F., et al. (1999). Disruption of the 12/15-lipoxygenase gene diminishes atherosclerosis in apo E-deficient mice. J. Clin. Invest. 103, 1597-1604. doi: 10.1172/JCI5897

Czimmerer, Z., Varga, T., Poliska, S., Nemet, I., Szanto, A., and Nagy, L. (2012). Identification of novel markers of alternative activation and potential endogenous PPAR $\gamma$ ligand production mechanisms in human IL-4 stimulated differentiating macrophages. Immunobiology 217, 1301-1314. doi: 10.1016/j. imbio.2012.08.270

Folcik, V. A., Nivar-Aristy, R. A., Krajewski, L. P., and Cathcart, M. K. (1995). Lipoxygenase contributes to the oxidation of lipids in human atherosclerotic plaques. J. Clin. Invest. 96, 504-510. doi: 10.1172/JCI118062

Fredman, G., Hellmann, J., Proto, J. D., Kuriakose, G., Colas, R. A., Dorweiler, B., et al. (2016). An imbalance between specialized pro-resolving lipid mediators and pro-inflammatory leukotrienes promotes instability of atherosclerotic plaques. Nat. Commun. 7, 12859. doi: 10.1038/ncomms12859

Gertow, K., Nobili, E., Folkersen, L., Newman, J. W., Pedersen, T. L., Ekstrand, J., et al. (2011). 12- and 15-lipoxygenases in human carotid atherosclerotic lesions: associations with cerebrovascular symptoms. Atherosclerosis 215, 411-416. doi: 10.1016/j.atherosclerosis.2011.01.015

Gundra, U. M., Girgis, N. M., Ruckerl, D., Jenkins, S., Ward, L. N., Kurtz, Z. D., et al. (2014). Alternatively activated macrophages derived from monocytes and tissue macrophages are phenotypically and functionally distinct. Blood 123 , e110-e122. doi: 10.1182/blood-2013-08-520619

Guo, Y., Zhang, W., Giroux, C., Cai, Y., Ekambaram, P., Dilly, A.-K., et al. (2011). Identification of the orphan $\mathrm{G}$ protein-coupled receptor GPR31 as a receptor for 12-(S)-hydroxyeicosatetraenoic acid. J. Biol. Chem. 286, 33832-33840. doi: 10.1074/jbc.M110.216564

Hammond, V. J., and O'Donnell, V. B. (2012). Esterified eicosanoids: generation, characterization and function. Biochim. Biophys. Acta 1818, 2403-2412. doi: 10.1016/j.bbamem.2011.12.013

Han, H., Xu, D., Liu, C., Claesson, H.-E., Björkholm, M., and Sjöberg, J. (2014). Interleukin-4-mediated 15-lipoxygenase-1 trans-activation requires UTX recruitment and H3K27me3 demethylation at the promoter in A549 cells. PLoS One 9, e85085. doi: 10.1371/journal.pone.0085085

Harats, D., Shaish, A., George, J., Mulkins, M., Kurihara, H., Levkovitz, H., et al. (2000). Overexpression of 15-lipoxygenase in vascular endothelium accelerates early atherosclerosis in LDL receptor-deficient mice. Arterioscler. Thromb. Vasc. Biol. 20, 2100-2105. doi: 10.1161/01.ATV.20.9.2100

Henson, P. M. (2017). Cell removal: efferocytosis. Annu. Rev. Cell Dev. Biol. 33, 127-144. doi: 10.1146/annurev-cellbio-111315-125315

Heydeck, D., Thomas, L., Schnurr, K., Trebus, F., Thierfelder, W. E., Ihle, J. N., et al. (1998). Interleukin-4 and -13 induce upregulation of the murine macrophage 12/15-lipoxygenase activity: evidence for the involvement of transcription factor STAT6. Blood 92, 2503-2510.

Heydeck, D., Upston, J. M., Viita, H., Ylä-Herttuala, S., and Stocker, R. (2001). Oxidation of LDL by rabbit and human 15-lipoxygenase: prevalence of nonenzymatic reactions. J. Lipid Res. 42, 1082-1088.

Huang, J. T., Welch, J. S., Ricote, M., Binder, C. J., Willson, T. M., Kelly, C., et al. (1999). Interleukin-4-dependent production of PPAR-gamma ligands in macrophages by 12/15-lipoxygenase. Nature 400, 378-382. doi: 10.1038/ 22572

Hultén, L. M., Olson, F. J., Aberg, H., Carlsson, J., Karlström, L., Borén, J., et al. (2010). 15-Lipoxygenase-2 is expressed in macrophages in human carotid plaques and regulated by hypoxia-inducible factor-1alpha. Eur. J. Clin. Invest. 40, 11-17. doi: 10.1111/j.1365-2362.2009.02223.x

Hutchins, P. M., and Murphy, R. C. (2012). Cholesteryl ester acyl oxidation and remodeling in murine macrophages: formation of oxidized phosphatidylcholine. J. Lipid Res. 53, 1588-1597. doi: 10.1194/jlr.M026799

Hutchins, P. M., Moore, E. E., and Murphy, R. C. (2011). Electrospray MS/MS reveals extensive and nonspecific oxidation of cholesterol esters in human peripheral vascular lesions. J. Lipid Res. 52, 2070-2083. doi: 10.1194/jlr. M019174

Ivanov, I., Kuhn, H., and Heydeck, D. (2015). Structural and functional biology of arachidonic acid 15-lipoxygenase-1 (ALOX15). Gene 573, 1-32. doi: 10.1016/j. gene.2015.07.073

Jack, G. S., Brash, A. R., Olson, S. J., Manning, S., Coffey, C. S., Smith, J. A., et al. (2000). Reduced 15-lipoxygenase-2 immunostaining in prostate adenocarcinoma: correlation with grade and expression in high-grade prostatic intraepithelial neoplasia. Hum. Pathol. 31, 1146-1154. doi: 10.1053/hupa. 2000.16670

Kühn, H., and Chan, L. (1997). The role of 15-lipoxygenase in atherogenesis: pro- and antiatherogenic actions. Curr. Opin. Lipidol. 8, 111-117. doi: 10.1097/00041433-199704000-00009 
Kühn, H., Belkner, J., Wiesner, R., Schewe, T., Lankin, V. Z., and Tikhaze, A. K. (1992). Structure elucidation of oxygenated lipids in human atherosclerotic lesions. Eicosanoids 5, 17-22.

Kühn, H., Belkner, J., Suzuki, H., and Yamamoto, S. (1994). Oxidative modification of human lipoproteins by lipoxygenases of different positional specificities. J. Lipid Res. 35, 1749-1759.

Kühn, H., Belkner, J., Zaiss, S., Fährenklemper, T., and Wohlfeil, S. (1994). Involvement of 15-lipoxygenase in early stages of atherogenesis. J. Exp. Med. 179, 1903-1911. doi: 10.1084/jem.179.6.1903

Kühn, H., Heydeck, D., Hugou, I., and Gniwotta, C. (1997). In vivo action of 15-lipoxygenase in early stages of human atherogenesis. J. Clin. Invest. 99, 888-893. doi: 10.1172/JCI119253

Kuhn, H., Banthiya, S., and van Leyen, K. (2015). Mammalian lipoxygenases and their biological relevance. Biochim. Biophys. Acta 1851, 308-330. doi: 10.1016/j. bbalip.2014.10.002

Kuhn, H., Gehring, T., Schröter, A., and Heydeck, D. (2016). Cytokinedependent expression regulation of ALOX15. J. Cytokine Biol. 1, 1-14. doi: 10.4172/2576-3881.1000106

Kuhn, H., Humeniuk, L., Kozlov, N., Roigas, S., Adel, S., and Heydeck, D. (2018). The evolutionary hypothesis of reaction specificity of mammalian ALOX15 orthologs. Prog. Lipid Res. 72, 55-74. doi: 10.1016/j.plipres.2018.09.002

Kutzner, L., Goloshchapova, K., Heydeck, D., Stehling, S., Kuhn, H., and Schebb, N. H. (2017). Mammalian ALOX15 orthologs exhibit pronounced dual positional specificity with docosahexaenoic acid. Biochim. Biophys. Acta Mol. Cell. Biol. Lipids 1862, 666-675. doi: 10.1016/j.bbalip.2017.04.001

Li, J., Rao, J., Liu, Y., Cao, Y., Zhang, Y., Zhang, Q., et al. (2013). 15-Lipoxygenase promotes chronic hypoxia-induced pulmonary artery inflammation via positive interaction with nuclear factor-кB. Arterioscler. Thromb. Vasc. Biol. 33, 971-979. doi: 10.1161/ATVBAHA.113.301335

Liu, C., Xu, D., Han, H., Fan, Y., Schain, F., Xu, Z., et al. (2012). Transcriptional regulation of 15-lipoxygenase expression by histone h3 lysine 4 methylation/ demethylation. PLoS One 7, e52703. doi: 10.1371/journal.pone.0052703

Lumbroso, D., Soboh, S., Maimon, A., Schif-Zuck, S., Ariel, A., and BurstynCohen, T. (2018). Macrophage-derived protein S facilitates apoptotic polymorphonuclear cell clearance by resolution phase macrophages and supports their reprogramming. Front. Immunol. 9, 358. doi: 10.3389/ fimmu.2018.00358

Magnusson, L. U., Lundqvist, A., Karlsson, M. N., Skålén, K., Levin, M., Wiklund, O., et al. (2012). Arachidonate 15-lipoxygenase type B knockdown leads to reduced lipid accumulation and inflammation in atherosclerosis. PLoS One 7, e43142. doi: 10.1371/journal.pone.0043142

Maskrey, B. H., Bermúdez-Fajardo, A., Morgan, A. H., Stewart-Jones, E., Dioszeghy, V., Taylor, G. W., et al. (2007). Activated platelets and monocytes generate four hydroxyphosphatidylethanolamines via lipoxygenase. J. Biol. Chem. 282, 20151-20163. doi: 10.1074/jbc.M611776200

Merched, A. J., Ko, K., Gotlinger, K. H., Serhan, C. N., and Chan, L. (2008). Atherosclerosis: evidence for impairment of resolution of vascular inflammation governed by specific lipid mediators. FASEB J. 22, 3595-3606. doi: 10.1096/fj.08-112201

Molofsky, A. B., Nussbaum, J. C., Liang, H.-E., Dyken, S. J. V., Cheng, L. E., Mohapatra, A., et al. (2013). Innate lymphoid type 2 cells sustain visceral adipose tissue eosinophils and alternatively activated macrophages. J. Exp. Med. 210, 535-549. doi: 10.1084/jem.20121964

Murray, P. J., Allen, J. E., Biswas, S. K., Fisher, E. A., Gilroy, D. W., Goerdt, S., et al. (2014). Macrophage activation and polarization: nomenclature and experimental guidelines. Immunity 41, 14-20. doi: 10.1016/j.immuni. 2014.06.008

Namgaladze, D., Snodgrass, R. G., Angioni, C., Grossmann, N., Dehne, N., Geisslinger, G., et al. (2015). AMP-activated protein kinase suppresses arachidonate 15-lipoxygenase expression in interleukin 4-polarized human macrophages. J. Biol. Chem. 290, 24484-24494. doi: 10.1074/jbc.M115.678243

Nassar, G. M., Morrow, J. D., Roberts, L. J., Lakkis, F. G., and Badr, K. F. (1994). Induction of 15-lipoxygenase by interleukin-13 in human blood monocytes. J. Biol. Chem. 269, 27631-27634.

O’Donnell, V. B., and Murphy, R. C. (2012). New families of bioactive oxidized phospholipids generated by immune cells: identification and signaling actions. Blood 120, 1985-1992. doi: 10.1182/blood-2012-04-402826
O’Donnell, V. B., Aldrovandi, M., Murphy, R. C., and Krönke, G. (2019). Enzymatically oxidized phospholipids assume center stage as essential regulators of innate immunity and cell death. Sci. Signal. 12, 1-12. doi: 10.1126/scisignal.aau2293

Paul, W. E. (2015). History of interleukin-4. Cytokine 75, 3-7. doi: 10.1016/j. cyto.2015.01.038

Poon, I. K. H., Lucas, C. D., Rossi, A. G., and Ravichandran, K. S. (2014). Apoptotic cell clearance: basic biology and therapeutic potential. Nat. Rev. Immunol. 14, 166-180. doi: 10.1038/nri3607

Rai, G., Joshi, N., Perry, S., Yasgar, A., Schultz, L., Jung, J. E., et al. (2013). “Discovery of ML351, a potent and selective inhibitor of human 15-lipoxygenase-1," in Probe Reports from the NIH Molecular Libraries Program (Bethesda (MD): National Center for Biotechnology Information (US)). Available at: http:// www.ncbi.nlm.nih.gov/books/NBK190602/ [Accessed May 13, 2019].

Rapp, J. H., Connor, W. E., Lin, D. S., Inahara, T., and Porter, J. M. (1983). Lipids of human atherosclerotic plaques and xanthomas: clues to the mechanism of plaque progression. J. Lipid Res. 24, 1329-1335.

Rong, S., Cao, Q., Liu, M., Seo, J., Jia, L., Boudyguina, E., et al. (2012). Macrophage $12 / 15$ lipoxygenase expression increases plasma and hepatic lipid levels and exacerbates atherosclerosis. J. Lipid Res. 53, 686-695. doi: 10.1194/jlr.M022723

Rothe, T., Gruber, F., Uderhardt, S., Ipseiz, N., Rössner, S., Oskolkova, O., et al. (2015). 12/15-Lipoxygenase-mediated enzymatic lipid oxidation regulates DC maturation and function. J. Clin. Invest. 125, 1944-1954. doi: 10.1172/JCI78490

Rydberg, E. K., Krettek, A., Ullström, C., Ekström, K., Svensson, P.-A., Carlsson, L. M. S., et al. (2004). Hypoxia increases LDL oxidation and expression of 15-lipoxygenase-2 in human macrophages. Arterioscler. Thromb. Vasc. Biol. 24, 2040-2045. doi: 10.1161/01.ATV.0000144951.08072.0b

Sadeghian, H., and Jabbari, A. (2016). 15-Lipoxygenase inhibitors: a patent review. Expert. Opin. Ther. Pat. 26, 65-88. doi: 10.1517/13543776.2016.1113259

Schewe, T., Halangk, W., and Hiebsch, C. Rapoport SM. (1975). A lipoxygenase in rabbit reticulocytes which attacks phospholipids and intact mitochondria. FEBS Lett. 60, 149-152. doi: 10.1016/0014-5793(75)80439-X

Schif-Zuck, S., Gross, N., Assi, S., Rostoker, R., Serhan, C. N., and Ariel, A. (2011) Saturated-efferocytosis generates pro-resolving CD11b low macrophages: modulation by resolvins and glucocorticoids. Eur. J. Immunol. 41, 366-379. doi: 10.1002/eji.201040801

Schweiger, D., Fürstenberger, G., and Krieg, P. (2007). Inducible expression of 15-lipoxygenase-2 and 8-lipoxygenase inhibits cell growth via common signaling pathways. J. Lipid Res. 48, 553-564. doi: 10.1194/jlr.M600311-JLR200

Sendobry, S. M., Cornicelli, J. A., Welch, K., Grusby, M. J., and Daugherty, A. (1998) Absence of $\mathrm{T}$ lymphocyte-derived cytokines fails to diminish macrophage 12/15-lipoxygenase expression in vivo. J. Immunol. 161, 1477-1482.

Shankaranarayanan, P., Chaitidis, P., Kühn, H., and Nigam, S. (2001). Acetylation by histone acetyltransferase CREB-binding protein/p300 of STAT6 is required for transcriptional activation of the 15-lipoxygenase-1 gene. J. Biol. Chem. 276, 42753-42760. doi: 10.1074/jbc.M102626200

Shappell, S. B., Boeglin, W. E., Olson, S. J., Kasper, S., and Brash, A. R. (1999). 15-lipoxygenase-2 (15-LOX-2) is expressed in benign prostatic epithelium and reduced in prostate adenocarcinoma. Am. J. Pathol. 155, 235-245. doi: 10.1016/ S0002-9440(10)65117-6

Shen, J., Herderick, E., Cornhill, J. F., Zsigmond, E., Kim, H. S., Kühn, H., et al. (1996). Macrophage-mediated 15-lipoxygenase expression protects against atherosclerosis development. J. Clin. Invest. 98, 2201-2208. doi: 10.1172/JCI119029

Shureiqi, I., Jiang, W., Zuo, X., Wu, Y., Stimmel, J. B., Leesnitzer, L. M., et al. (2003). The 15-lipoxygenase-1 product 13-S-hydroxyoctadecadienoic acid downregulates PPAR-delta to induce apoptosis in colorectal cancer cells. Proc. Natl. Acad. Sci. U.S.A. 100, 9968-9973. doi: 10.1073/pnas. 1631086100

Sigal, E., Grunberger, D., Highland, E., Gross, C., Dixon, R. A., and Craik, C. S. (1990). Expression of cloned human reticulocyte 15-lipoxygenase and immunological evidence that 15-lipoxygenases of different cell types are related. J. Biol. Chem. 265, 5113-5120.

Singh, N. K., and Rao, G. N. (2019). Emerging role of 12/15-Lipoxygenase (ALOX15) in human pathologies. Prog. Lipid Res. 73, 28-45. doi: 10.1016/j. plipres.2018.11.001

Snodgrass, R. G., Zezina, E., Namgaladze, D., Gupta, S., Angioni, C., Geisslinger, G., et al. (2018). A novel function for 15-lipoxygenases in cholesterol homeostasis and CCL17 production in human macrophages. Front. Immunol. 9, 1906. doi: 10.3389/fimmu.2018.01906 
Spite, M., Clària, J., and Serhan, C. N. (2014). Resolvins, specialized proresolving lipid mediators, and their potential roles in metabolic diseases. Cell Metab. 19, 21-36. doi: 10.1016/j.cmet.2013.10.006

Stables, M. J., Shah, S., Camon, E. B., Lovering, R. C., Newson, J., Bystrom, J., et al. (2011). Transcriptomic analyses of murine resolution-phase macrophages. Blood 118, e192-e208. doi: 10.1182/blood-2011-04-345330

Suraneni, M. V., Schneider-Broussard, R., Moore, J. R., Davis, T. C., Maldonado, C. J., $\mathrm{Li}, \mathrm{H}$., et al. (2010). Transgenic expression of 15-lipoxygenase 2 (15-LOX2) in mouse prostate leads to hyperplasia and cell senescence. Oncogene 29, 42614275. doi: 10.1038/onc.2010.197

Suraneni, M. V., Moore, J. R., Zhang, D., Badeaux, M., Macaluso, M. D., DiGiovanni, J., et al. (2014). Tumor-suppressive functions of 15-lipoxygenase-2 and RB1CC1 in prostate cancer. Cell Cycle 13, 1798-1810. doi: 10.4161/cc.28757

Tang, S., Bhatia, B., Maldonado, C. J., Yang, P., Newman, R. A., Liu, J., et al. (2002). Evidence that arachidonate 15-lipoxygenase 2 is a negative cell cycle regulator in normal prostate epithelial cells. J. Biol. Chem. 277, 16189-16201. doi: 10.1074/jbc.M111936200

Tang, S., Bhatia, B., Zhou, J., Maldonado, C. J., Chandra, D., Kim, E., et al. (2004). Evidence that $\mathrm{Sp} 1$ positively and Sp3 negatively regulate and androgen does not directly regulate functional tumor suppressor 15-lipoxygenase 2 (15-LOX2) gene expression in normal human prostate epithelial cells. Oncogene 23, 69426953. doi: 10.1038/sj.onc. 1207913

Tang, D. G., Bhatia, B., Tang, S., and Schneider-Broussard, R. (2007). 15-lipoxygenase 2 (15-LOX2) is a functional tumor suppressor that regulates human prostate epithelial cell differentiation, senescence, and growth (size). Prostaglandins Other Lipid Mediat. 82, 135-146. doi: 10.1016/j.prostaglandins.2006.05.022

Uderhardt, S., Herrmann, M., Oskolkova, O. V., Aschermann, S., Bicker, W., Ipseiz, N., et al. (2012). 12/15-Lipoxygenase orchestrates the clearance of apoptotic cells and maintains immunologic tolerance. Immunity 36, 834-846. doi: 10.1016/j.immuni.2012.03.010

Uderhardt, S., Ackermann, J. A., Fillep, T., Hammond, V. J., Willeit, J., Santer, P., et al. (2017). Enzymatic lipid oxidation by eosinophils propagates coagulation, hemostasis, and thrombotic disease. J. Exp. Med. 214, 2121-2138. doi: 10.1084/ jem. 20161070

Varol, C., Mildner, A., and Jung, S. (2015). Macrophages: development and tissue specialization. Annu. Rev. Immunol. 33, 643-675. doi: 10.1146/ annurev-immunol-032414-112220

Weibel, G. L., Joshi, M. R., Alexander, E. T., Zhu, P., Blair, I. A., and Rothblat, G. H. (2009). Overexpression of human 15(S)-lipoxygenase-1 in RAW macrophages leads to increased cholesterol mobilization and reverse cholesterol transport. Arterioscler. Thromb. Vasc. Biol. 29, 837-842. doi: 10.1161/ATVBAHA.109.186163

Wenzel, S. E., Tyurina, Y. Y., Zhao, J., St Croix, C. M., Dar, H. H., Mao, G., et al. (2017). PEBP1 wardens ferroptosis by enabling lipoxygenase generation of lipid death signals. Cell 171, 628-641.e26. doi: 10.1016/j.cell.2017.09.044

Werner, M., Jordan, P. M., Romp, E., Czapka, A., Rao, Z., Kretzer, C., et al. (2019). Targeting biosynthetic networks of the proinflammatory and proresolving lipid metabolome. FASEB J. 33, 6140-6153. doi: 10.1096/fj.201802509R

Werz, O., Gerstmeier, J., Libreros, S., De la Rosa, X., Werner, M., Norris, P. C., et al. (2018). Human macrophages differentially produce specific resolvin or leukotriene signals that depend on bacterial pathogenicity. Nat. Commun. 9, 59. doi: 10.1038/s41467-017-02538-5

Wuest, S. J. A., Crucet, M., Gemperle, C., Loretz, C., and Hersberger, M. (2012). Expression and regulation of 12/15-lipoxygenases in human primary macrophages. Atherosclerosis 225, 121-127. doi: 10.1016/j.atherosclerosis. 2012.07.022

Yamanishi, Y., and Karasuyama, H. (2016). Basophil-derived IL-4 plays versatile roles in immunity. Semin. Immunopathol. 38, 615-622. doi: 10.1007/ s00281-016-0568-y

Yang, W., Zhao, X., Tao, Y., Wu, Y., He, F., and Tang, L. (2019). Proteomic analysis reveals a protective role of specific macrophage subsets in liver repair. Sci. Rep. 9, 2953. doi: 10.1038/s41598-019-39007-6

Zhang, W., Zhong, W., Sun, Q., Sun, X., and Zhou, Z. (2017). Hepatic overproduction of 13-HODE due to ALOX15 upregulation contributes to alcohol-induced liver injury in mice. Sci. Rep. 7, 8976. doi: 10.1038/s41598-017-02759-0

Zuo, X., Wu, Y., Morris, J. S., Stimmel, J. B., Leesnitzer, L. M., Fischer, S. M., et al. (2006). Oxidative metabolism of linoleic acid modulates PPAR-beta/delta suppression of PPAR-gamma activity. Oncogene 25, 1225-1241. doi: 10.1038/ sj.onc. 1209160

Conflict of Interest Statement: The authors declare that the research was conducted in the absence of any commercial or financial relationships that could be construed as a potential conflict of interest.

Copyright (c) 2019 Snodgrass and Brüne. This is an open-access article distributed under the terms of the Creative Commons Attribution License (CC BY). The use, distribution or reproduction in other forums is permitted, provided the original author(s) and the copyright owner(s) are credited and that the original publication in this journal is cited, in accordance with accepted academic practice. No use, distribution or reproduction is permitted which does not comply with these terms. 